\title{
Evaluation of Line of Balance Method In View Of Hidden Idle Times and Their Costs (Case Study: Housing Units in Iraq)
}

\author{
Aqeel Abdulhasan Hussein ${ }^{1}$, Ali Majdi ${ }^{1}$ \\ ${ }^{1}$ Al-Mustaqbal University College \\ Al-Najaf Street, Hilla, Iraq \\ aqeelabdulhassan@ mustaqbal-college.ed.iq; alimajdi@mustaqbal-college.ed.iq
}

\begin{abstract}
This study is dealing with scheduling of repetitive construction projects by using Line of Balance (LOB) method and taking a construction project as a case study. The project is consisting of 100 identical housing units. LOB is used in scheduling repetitive construction projects and it is suitable for medium to long-range scheduling. It is resource-based technique. This means ensuring continuity of work and efficient use of resources. The optimum number of crews is selected so that all crews will perform the same number of units in the same period of time. This approach is essentially the line of balance concept. It is shown in this study that the LOB method is easy method in scheduling repetitive projects in that it is easy to obtain graphically the needed data such as start or finish of each activity. The disadvantage of LOB method is that there is a need to adjust the resulted graph to take in account delays in start times resulting from several reasons. One reason is to take in account the differences between the numbers of units assigned to the work gangs. Another is to take in account the constraint of continuity. Making these adjustments leads to additional costs resulted from hidden idle times and from lengthening the duration of the whole project. One of the main objectives in scheduling repetitive projects is to minimize idle times that is to make resources working without many interruptions. In this study the resulting cost from adjustment reached about $4 \%$ from the total cost of the project. There is ability to make an alternative LOB graph that has compressed work and gets less period and less cost of project in the same time. Further research work is recommended to get such LOB scheduling.
\end{abstract}

Keywords: Scheduling, Repetitive Projects, Critical Path Method (CPM), Line of Balance (LOB), Idle Times, Direct Costs, Indirect Costs.

\section{Introduction}

Repetitive activities are found usually in the construction projects such as housing development projects. In this type of projects, construction crews are often required to execute the same work in various locations of the project, moving from one location to another. Due to this crew movement, available scheduling methods for repetitive construction projects try to make work continuity by enabling each crew to finish work in one location of the project and move to the next without interruption. The Critical Path Method (CPM) cannot assure this requirement because only technical precedence and resource availability constraints are explicitly shown in CPM networks. The line Of Balance method (LOB) described in this research is more suitable to ensure continuous resource usage [1].

This research tries to investigate the efficiency LOB as a method in repetitive scheduling. The research will focus on a real project which is consisting of 100 housing units. The guideline concept of this research is that the basic requirement in any method of scheduling repetitive construction projects like executing 100 housing units is to take in account the balance condition in order to insure suitable number of work gangs to satisfy both compliance and continuity requirements. However, idle times usually exist and lead to elongate the project duration and increase its cost. In usual use of LOB method these idle times are not visible. This research highlights this problem and give a clearer picture to the resulted scheduling.

\section{The Research Aims}

The main aim of this research is to introduce the application of the Line of Balance Scheduling Method (LOB) and related tools on a repetitive construction project that is the project of 100 housing units. and to explain the disadvantages of direct use of LOB method regarding the inherent hidden idle times in scheduling projects having repetitive nature such as executing large number of housing units. 


\section{The problems of delay and balance}

There are two main constraints that control the work sequence of resources, technological precedence and resource availability. In figure (1) there are two diagrams for a repetitive project consisting of 3 units, each one is requiring 3 activities (A, B and C). As shown in part (a) from the figure there are lags between activities. This means that resource availability constraints are satisfied, but technological constraints are not. This period of waiting is called "idle time". One of the main objectives in scheduling repetitive projects is to minimize idle time that is to make resources working without interruption. Contractors usually pay for resources from their arriving to the site, until they finish work and leave. Therefore, it is important to minimize idle time and hence avoid extra costs [2]. This may be achieved by delaying activity's' start dates as it is shown in part (b) where the total project idle time has been eliminated without changing project duration. However it sometimes happens that it is impossible to make this without some increase in project duration. Optimization methods deals with this problem trying to reach a minimum duration for the project and in the same time a minimum cost of delay in individual activities [3].

Another issue regarding scheduling repetitive projects is how to make decision regarding the number of gangs' i.e the crew of work. In other words: how many units should be executed (for a certain activity) during every certain period. The term Balance is dealing with this problem.

\section{LOB Approach}

LOB is used in scheduling repetitive construction projects and it is suitable for medium to long-range scheduling. It is resource-based technique. This means ensuring continuity of work and efficient use of resources. Construction of one unit is broken into activities or groups of activities. Crews of workers perform the tasks associated with these activities. The project is then constructed by arranging the work of each crew to proceed continuously from the first to the last unit. If individual activities in each repetitive unit have the same rate of work, they are arranged to progress one after the other from the first to the last unit. This is illustrated in Figure (2). A gap between individual activities is a buffer zone, which is determined by applying logic constraints and taking a time contingency of each activity [4].

When scheduling is based on construction units, fairly repetitive construction subdivisions can be developed and same work rhythm for all the involved crews will be assumed. The number of optimum crews is selected so that all crews will perform the same number of units in the same period of time. This approach is essentially the line of balance concept [5].

A uniform rate of progress of individual activities is rarely achieved. A more realistic is the variation from activity to activity. Activities of such varying rates of progress are then linked together so that continuity of work of each crew is maintained. In LOB the duration of each activity is to be calculated first and then to be plotted on a chart representing the tool of the project monitoring. This means that the chart is extracted as a result of the analysis, while in other methods like RSM the chart leads the analyst to conclude the overall duration [3]. 

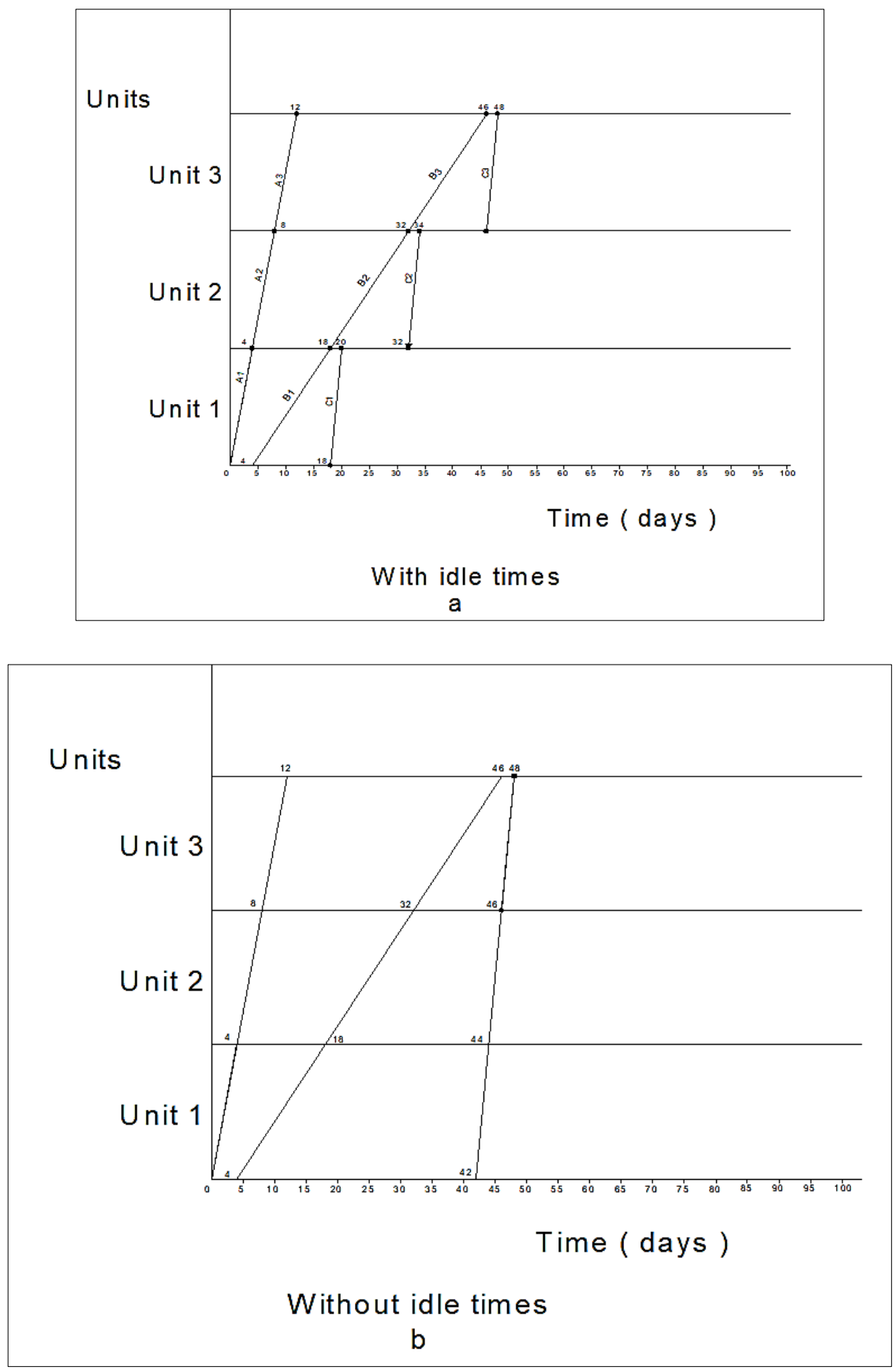

Fig. 1: Repetitive Project Example. 


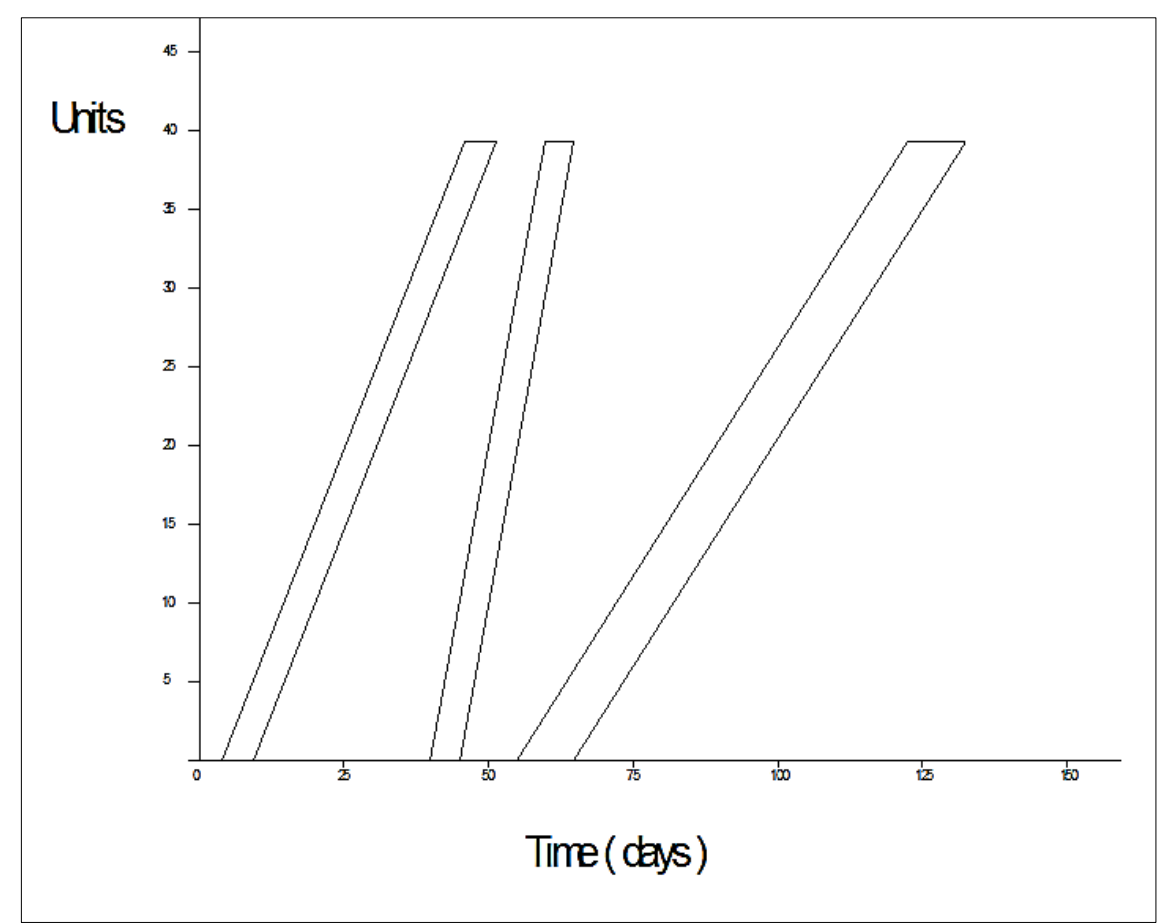

Fig. 2: Graph of LOB Technique.

The unit production rate $(\mathrm{R})$ is the number of repetitive units that can be completed by a resource during a unit of time. This rate should be the same for all activities (even it would be so approximately). The size of work (M) for each activity (for only one unit) is expressed in (man. hour) unit which is resulted from dividing the quantity of work by the productivity. For example if the quantity of painting is $200 \mathrm{sq}$. $\mathrm{m}$ and the painter makes in one hour $5 \mathrm{sq} . \mathrm{m}$, then the size of painting work will be $200 / 5=40$ man.hr. The number of workers (H) (resources in general) available for each unit depends on the capability of the firm or the contractor and it is set as given data for calculation. Other given data is the number of working days in a week (dw) and so is the number of working hours in a week (hw). Then, the number of workers $\mathrm{G}$ needed in all gangs can be calculated from following equation:

$$
G=M \times R / h w
$$

For example, if M equals 40 man.hr and there will be $42 \mathrm{hrs}$ in a week and 6 working days in a week and the required rate of production is 5 units/ week then the number of all workers is:

$$
\mathrm{G}=40 \times 5 / 42=4.76 \text { workers say } 5 \text { workers }
$$

Now, if the size of one gang $(\mathrm{H})$ is 2 workers then the manager should decide whether there would be 6 workers as whole instead of 5 (this makes 3 gangs and the rate $\mathrm{R}$ will be more than planned) or 4 workers as whole instead of 5 (this makes 2 gangs and the rate $\mathrm{R}$ will be less than planned). The selected number however is termed as (S) while the calculated number is termed as $(\mathrm{G})$ as seen above.

The duration of the activity for each unit (D) can be calculated from:

$$
D=M /(H \times h d)
$$

Where hd is the working hours per day and can be calculated from:

$$
h d=h w / d w
$$


For example the duration of painting will be: $40 /(2 \times 42 / 6)=2.86$ days. On the other hand, the total period of activity for entire project $(\mathrm{T}) \mathrm{b}$ can be calculated from:

$$
T=(N-1) \times d w / R^{\prime}
$$

Where $\mathrm{N}$ is the number of units in the entire project and $\mathrm{R}^{\prime}$ is the new rate of production and can be calculated from:

$$
R^{\prime}=R \times S / G
$$

For example, the total period of painting for entire project (of 100 units) will be:

$$
\mathrm{R}^{\prime}=6 \times 5 / 6=5 \text { units/wk. }
$$

$$
\mathrm{T}=99 \times 6 / 5=118.8 \text { days }
$$

If the quantity of work in any activity is the same for the sequence units then the production line will be straight line having one constant slope. The production lines may be as segments or connected lines if the units have different quantities of work along the sequence (this is the common situation) [6].

\subsection{Drawing conventions}

A small number of units would not be found in realistic projects like that of the research which involves a large number of units. There are some conventional representations may be adopted. To explain some of these conventions, the LOB diagram shown in Figure (2) shows the balance lines for three activities. Each activity is represented as a strip has a width equals to the duration (D) and a slope equals to the rate of production (R) [6].

There is usually a difference between two cases during preparation of the BOL diagram. First case is the theoretical results as computed using the procedure and equations illustrated in the previous article. Second case is an adjusted one that prepared for taking in account the following:

1. Integer numbers must be used to assign start and finish of each activity

2. The activities are grouped according to the number of gangs and there are delay times resulting from logical sequences.

3. There is ability to compress durations of activities by increasing resources.

\section{Project of Study}

The project under study is a housing projects consisting of 100 identical housing units. The manager is going to prepare groups (crews) so that each group should execute same number of units for each activity. The rate of production is decided to be one unit per working week. Each working week includes 40 working hours and 5 working days. As long as all units has identical activities, there is no meaning to choose one of them in priority of the others, so the 100 units will be numbered from unit 1 to unit 100. Table (1) shows the activities of a standard housing unit. There are 6 activities listed together with their symbols for easy reference.

Table 1: Activities of the Project.

\begin{tabular}{|l|l|l|l|l|l|}
\hline Activity & Symbol & $\begin{array}{l}\text { Size of Work } \\
(\mathrm{M}) \text { in (man.hr) }\end{array}$ & $\begin{array}{l}\text { Number of } \\
\text { Workers (H) }\end{array}$ & $\begin{array}{l}\text { Cost in } \\
\$ 1000\end{array}$ & $\begin{array}{l}\text { Cost of Delay } \\
\text { in \$1000/day }\end{array}$ \\
\hline Foundation & FND & 288 & 1 & 39 & 8 \\
\hline Structure & STR & 328 & 1 & 84 & 28 \\
\hline Masonry & MAS & 704 & 2 & 30 & 7 \\
\hline $\begin{array}{l}\text { Internal } \\
\text { Works }\end{array}$ & INT & 1792 & 2 & 200 & 70 \\
\hline Fencing & FNC & 816 & 2 & 30 & 8 \\
\hline Finishing & FSH & 800 & 10 & 25 & 6 \\
\hline
\end{tabular}


An important feature here is the grouping of the main activities. All activities are grouped such that they are in series connection (one after one). During the execution these groups may be exploded into what a level of detail needed giving information for other production control tools (short term plans). This is the same situation when using any method for scheduling $[7,8]$.

\section{LOB Diagram}

The calculations had been made to obtain the number of workers in each gang $(\mathrm{G})$, the adjusted number of workers $(\mathrm{S})$, the unit duration (D), the adjusted rate of production and the total period of each activity (T). The results are shown in Table (2).

Table 2: Results of LOB calculations.

\begin{tabular}{|c|l|l|l|l|l|l|}
\hline Activity & $\begin{array}{l}\text { Number of } \\
\text { Workers } \\
(\mathrm{G})\end{array}$ & $\begin{array}{l}\text { Adjusted } \\
\text { Number of } \\
\text { Workers (S) }\end{array}$ & $\begin{array}{l}\text { Number of } \\
\text { Units For } \\
\text { Each Gang }\end{array}$ & $\begin{array}{l}\text { Adjusted Rate } \\
\text { of Production } \\
\left(\mathrm{R}^{\prime}\right)\end{array}$ & $\begin{array}{l}\text { Unit } \\
\text { Duration In } \\
\text { days (D) }\end{array}$ & $\begin{array}{l}\text { Total Period } \\
\text { In days (T) }\end{array}$ \\
\hline FND & 7.2 & 8 & 8 & 1.111 & 36 & 445.5 \\
\hline STR & 8.2 & 9 & 9 & 1.098 & 41 & 450.8 \\
\hline MAS & 17.6 & 18 & 9 & 1.023 & 44 & 483.9 \\
\hline INT & 44.8 & 46 & 23 & 1.027 & 112 & 482.0 \\
\hline FNC & 20.4 & 22 & 11 & 1.078 & 51 & 459.2 \\
\hline FSH & 20 & 20 & 2 & 1.000 & 10 & 495 \\
\hline
\end{tabular}

Figure (3) shows the resulted LOB graph which represents the entire project. The total period of the project as seen in the figure is $\mathbf{8 1 5}$ days. With a daily indirect cost of $\$ \mathbf{5 0 0}$ the total indirect cost will be:

$$
500 \times 815=\$ \mathbf{4 0 7 , 5 0 0}
$$

The total direct cost of the project can be obtained as following:

$$
\text { Direct Cost }=\sum \text { Activity direct cost }=\$ \mathbf{4 0 , 8 0 0 , 0 0 0}
$$

The total cost of the project will be:

$$
\begin{gathered}
\text { Total Cost of Project }=\text { Direct Cost }+ \text { Indirect Cost } \\
=40,800,000+407,500=\$ \mathbf{4 1 , 2 0 7 , 5 0 0}
\end{gathered}
$$

\subsection{Adjusted LOB Diagram}

It can be shown from the previous articles that there is a need to make adjustment to the results. This adjustment includes delays for activities because of the following reasons:

1. Adjustment for the dates of start or finish to make integer figures. For example, the gang of foundation works (FND) for the first eight units finishes the work in 40.5 days and this should be adjusted to 41 days

2. Taking in account the differences between the numbers of units assigned to the work gangs, for example the gang of (STR) for the units (90 through 99) should delay the work for 9 days to make the start in the same time with the finish of the previous activity (FND)

3. Taking in account the constraint of continuity. For example, the rate of production for (FNC) is 1.078 and this is greater than that of the previous activity (INT) which is 1.027. This makes delays in (FNC) start times. For example, a delay of 3 days for start of units (77 through 88).

Figure (4) gives the adjusted LOB diagram. 


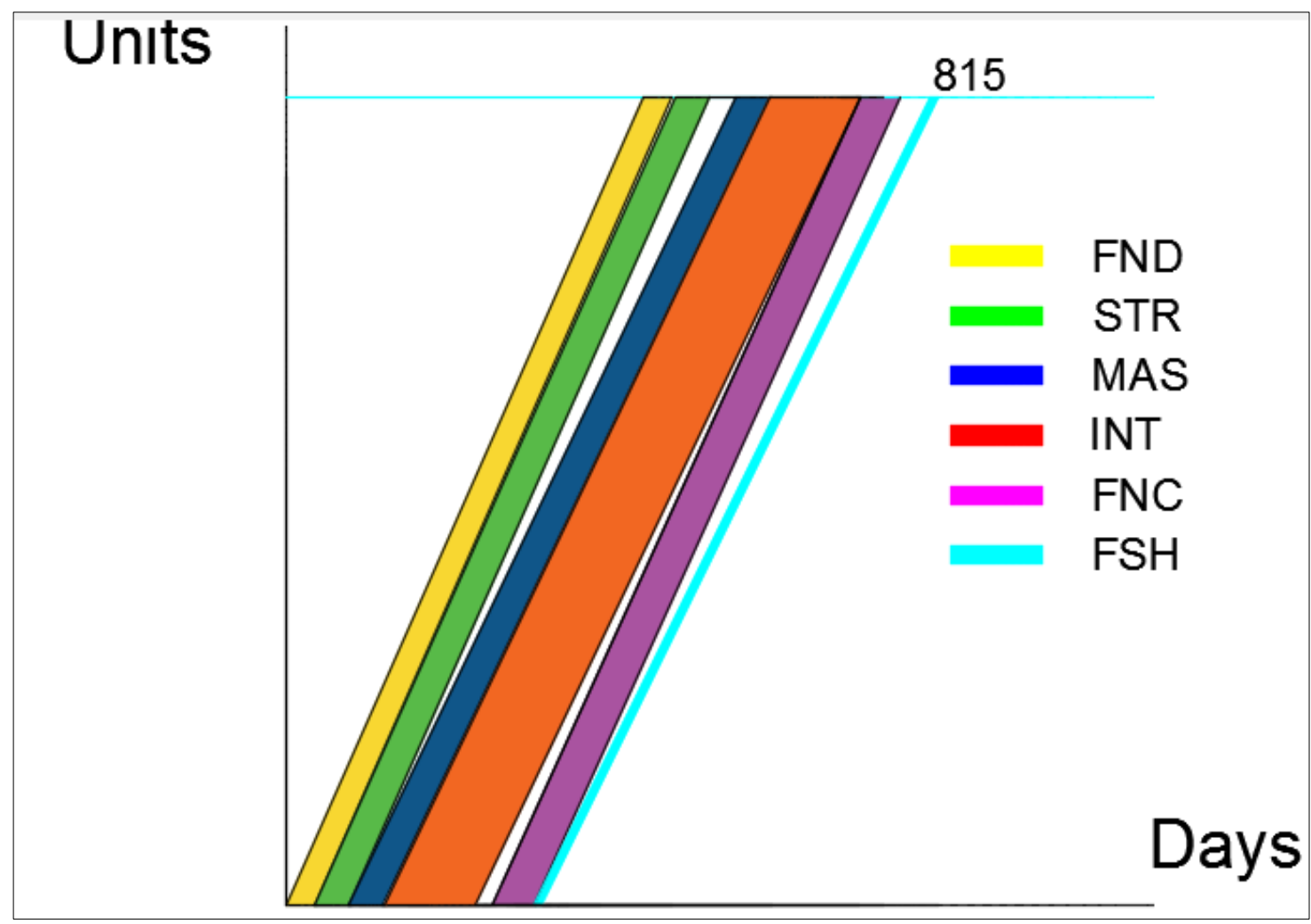

Fig. 3: Resulted LOB Diagram.

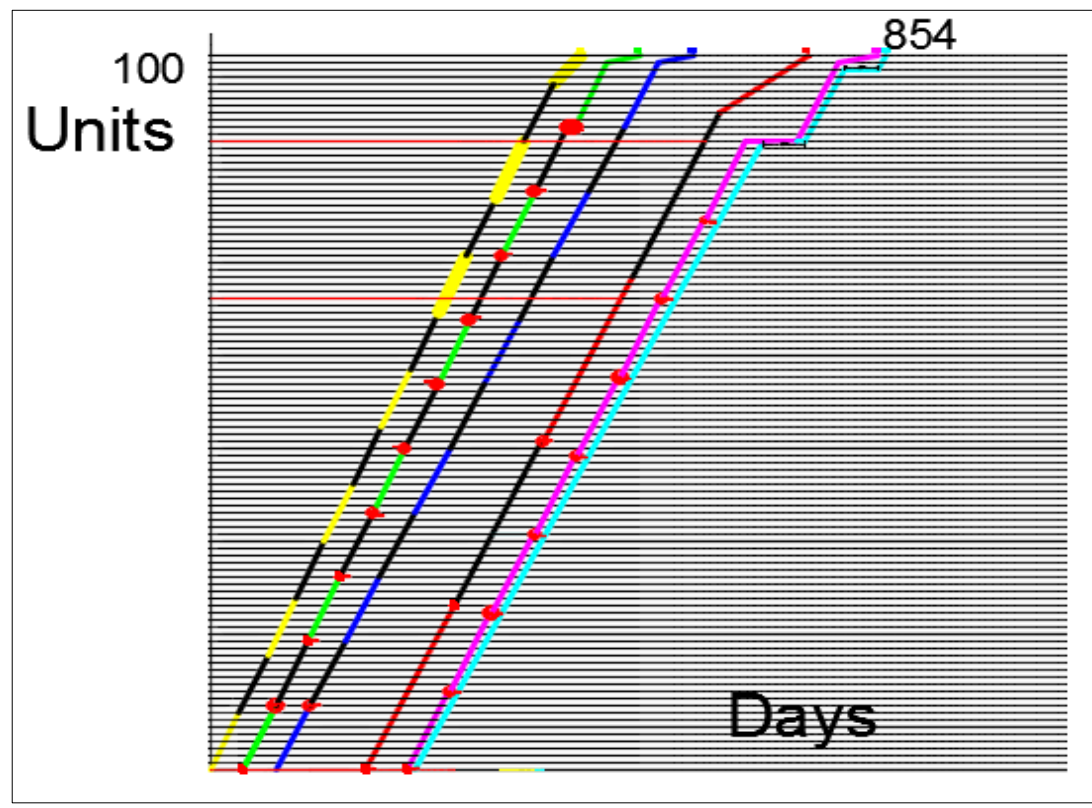

Fig. 4: Adjusted LOB Diagram.

Table (3) gives the total idle (delay) times and their costs resulting from adjustments for all activities involved in the project. 
Table 3: Idle (delay) times and their costs.

\begin{tabular}{|l|l|l|}
\hline Activity & $\begin{array}{l}\text { Total Idle Times in } \\
\text { days }\end{array}$ & $\begin{array}{l}\text { Cost of Idle Times } \\
\text { in } \$ 1000\end{array}$ \\
\hline FND & 0 & 0 \\
\hline STR & 9 & 252 \\
\hline MAS & 0 & 0 \\
\hline INT & 1 & 70 \\
\hline FNC & 85 & 680 \\
\hline FSH & 95 & 570 \\
\hline
\end{tabular}

The total period of the project as seen in the Figure (4) is 854 days. With a daily indirect cost of $\$ 500$ the total indirect cost will be:

$$
500 \times 854=\$ \mathbf{4 2 7 , 0 0 0}
$$

The total direct costs are same as before: $\$ \mathbf{4 0 , 8 0 0 , 0 0 0}$

This is so because the direct costs are related to the activities and unchanged when the idle times exist [7].

The total idle times cost from table (3) is $\mathbf{\$ 1 , 5 7 2 , 0 0 0}$

The total cost for entire project is $427000+40800000+1572000=\mathbf{\$ 4 2 , 7 9 9 , 0 0 0}$

This is more than the initial LOB schedule by: $\mathbf{4 \%}$

It is important to mention here that there is always ability to compress durations for activities by increasing resources and hence get a smaller duration for the total project $[9,10]$.

However, this alternative is out of the scope of this study and it is recommended to get such LOB scheduling

\section{Conclusion}

The LOB method is easy graphical method in scheduling repetitive projects in that it is easy to obtain all data needed such as start or finish of each activity and the expected complete activities (or units) in a certain time, all that is in one graph which is not the case in other scheduling methods .However, the disadvantage of LOB method as it is seen from this study is that there is a need to adjust the diagram to take in account delays in start times resulting from several reasons. In this study the resulting cost from adjustment reached about $4 \%$ from the total cost of the project.

\section{References}

[1] P. Lumsden, "The line-of-balance method," Pergamon Press Limited, 1968.

[2] C. Srisuwanrat, and Photios G. Ioannou, "Optimal scheduling of probabilistic repetitive projects using completed unit and genetic algorithms," in Proceedings of the 39th conference on Winter simulation: 40 years! The best is yet to come, pp. 2151-2158. IEEE Press, 2007.

[3] Robert B. Harris and Photios G. Ioannou, "CENTER FOR CONSTRUCTION ENGINEERING AND MANAGEMENT," (1998). REPETITIVE SCHEDULING METHOD," Civil and Environmental Engineering Department UNIVERSITY OF MICHIGAN Ann Arbor, Michigan, pp.UMCEE Report No. 98-35. [Online]. Available at: http://www.cem.umich.edu/ioannou/Pubs/CEE98_RSM/PGI_CEE98_RSM.pdf

[4] V. González, L. F. Alarcón, and P. Gazmuri., "Design of work in process buffers in repetitive building projects: a case study," in 14th annual conference of international group for lean construction, Proceedings IGLC-14, Santiago, Chile. 2006. 
[5] S. L. Kemmer, L. F. M. Heineck, and T. D. C. Alves, Thaís da C. L., "Using the Line of Balance for Production System Design" in Proc. 16th Annual Conf. of the Intl. Group for Lean Construction People, Culture and Change, Michigan, USA, 2010.

[6] J. Kankainen, and O. Seppänen, "A line-of-balance based schedule planning and control system," in 11th Annual conference on Lean Construction, Virginia, USA. pp. 22-24. 2003.

[7] Bennett, F. Lawrence, "The Management of Construction," University of Alaska, 2003.

[8] S. W. Nunnaly, "Construction Methods and Management," Pearson, 2007.

[9] Uher, E. Thomas, "Programming and scheduling techniques", University of New South Wales, 2003.

[10] Stevenson, William J., "Production/Operations Management", Irwin, 1996. 